\title{
Tiredness in Acute and Chronic Depression Treated with Transcranial Magnetic Stimulation
}

\author{
Saxby Pridmore \\ s.pridmore@utas.edu.au \\ School of Medicine, University of Tasmania, \\ Hobart, Tasmania, Australia; \\ TMS Department, Saint Helen's Hospital, \\ Hobart, Tasmania, Australia \\ (corresponding author) \\ Sheila Erger \\ Sheila.Erger@healthscope.com.au \\ TMS Department, Saint Helen's Hospital, \\ Hobart, Tasmania, Australia \\ Marzena Rybak \\ mrybak@netspace.net.au \\ TMS Department, Saint Helen's Hospital, \\ Hobart, Tasmania, Australia \\ Tamara May \\ Tamara.May@monash.edu \\ Department of Paediatrics and Education Research, \\ Monash University, Clayton, Australia
}

\begin{abstract}
Background: Tiredness is used in some characterizations of major depressive disorder (MDD). Transcranial magnetic stimulation (TMS) lowers all symptoms of MDD. Objective: To explore whether, 1) a visual analogue scale (VAS) for tiredness is a valid and reliable measure of a feature of MDD, and 2) TMS treatment reduces subjective tiredness occurring in MDD. Method: A naturalistic study of treatment with $10 \mathrm{~Hz}$ TMS. Completed pre- and post-treatment: HAMD-6, a visual analogue scale (VAS-6), the Clinical Global Impression - Severity (CGI-S) and a 'VAS-tiredness'. Two groups received TMS. Acute course: N=52 participants suffering acute MDD, received 20 treatment courses (total courses 86). Relapse prevention (RP) course: $\mathrm{N}=26$ participants suffering chronic relapsing MDD received scheduled episodic courses over 3 days; (total courses 266). VAS-tiredness scores were compared with the standardized tool results. Results: There were significant medium to large correlations between pre- and post-treatment VAS-tiredness and the standard depression measures (HAMD-6 .406 to .447, VAS-6 .446 to .525, CGI-S .348 to .407 ; all $p<.001)$. TMS treatment produced a significant reduction in VAS-
\end{abstract}


tiredness in both (Acute course and RP) groups (main effect: $F(1,350)=147.3$, $p<.001, \eta^{2}=.30$ ). The two groups displayed difference in the pre-treatment VAStiredness with the Acute group having higher scores pre-treatment. Post-treatment tiredness scores were similar. Conclusion: VAS-tiredness is a valid measure of a feature of MDD. VAS-tiredness provides potentially useful information and complements standard mood tools. TMS treatment can reduce tiredness in MDD.

Keywords: VAS-tiredness; MDD; TMS treatment

How to cite: Pridmore, Saxby, Sheila Erger, Marzena Rybak, and Tamara May (2019). "Tiredness in Acute and Chronic Depression Treated with Transcranial Magnetic Stimulation," American Journal of Medical Research 6(2): 7-18. doi:10.22381/AJMR6220191

Received 27 June 2019 • Received in revised form 6 August 2019

Accepted 6 August 2019• Available online 8 August 2019

\section{Introduction}

Tiredness is defined by English dictionaries as, "The state of wishing to sleep or rest" and has various synonyms. In clinical research a distinction has been attempted between tiredness ('natural tiredness') and fatigue ('unnatural tiredness') (Grape et al., 2017), but has not been widely embraced. The term fatigue occurs in discussions of somatic disease such as multiple sclerosis and rheumatoid arthritis. A systematic review of studies of people presenting to general practice complaining of tiredness (Stadje et al., 2016) found serious somatic disease was rare and the most the common diagnosis was depression $(18 \%)$.

Silverstein (1999) described "somatic depression" - a variation more common in females, characterized by fatigue and appetite and sleep disturbances. Vaccarino et al. (2008) report that 'feeling fatigued, weak and tired all over' is the most common somatic symptom of major depressive disorder (MDD), with $78 \%$ of patients reporting moderate or higher levels. Crowe et al. (2018) used the Experience Sampling Method to capture the within-day dynamics of various features of people with MDD and healthy controls. The MDD group experienced a significantly different pattern of tiredness - displaying a flatter course throughout the day. Chu et al. (2019) confirm tiredness and related sleep issues are possible symptoms of inflammation-related depression and are used as markers of response to anti-inflammatory medication.

The persistence of isolated symptoms following the treatment of MDD has been described (Katon et al., 1999). Ghanean et al. (2018) report that after treatment for depression, 10-35\% of patients continue to experience fatigue, and suggest possible pharmacological mitigation options. The impact of Transcranial Magnetic Stimulation (TMS) on subjective tiredness in MDD has received little attention.

Tiredness does not appear as a heading in the 17 item Hamilton Depression Rating Scale (HAMD-17) (Hamilton, 1960) or the Positive and Negative 
Affect Scale (Crawford and Henry, 2004). Lassitude is one of 10 diagnostic criteria of the Montgomery-Asberg Depression Rating Scale (Montgomery and Åsberg, 1979), low energy is one of 20 items listed in the Cornell Dysthymia Rating Scale (Cohen, 1997), and fatigue or loss of energy is one of 9 criteria of MDD in the 5th edition of the Diagnostic and Statistical Manual (Association, 2013). Tiredness was one of three primary symptoms of MDD in the 10th edition of International Classification of Diseases (Organization, 1992). It has less prominence in the ICD-11 but remains as part of the neurovegetative symptom cluster (Organization, 2018). Feeling tired or having little energy is one of 9 criteria in the subjective Patient Health Questionnaire depression module (Kroenke et al., 2001). Thus, tiredness and synonyms are measured by many but not all mood assessment tools.

We have used the HAMD-6 in a series of studies of TMS in the treatment of MDD (Pridmore et al., 2018; May and Pridmore, 2019). HAMD-6 criterion 2 'work and activities' scores feelings of 'fatigue or weakness' as a lowseverity feature. Criterion 3 'somatic symptoms' scores 'loss of energy and fatigability' as a mid-level severity feature. Criterion 6 'retardation' is an observational assessment of motor activity - the relationship of observed retardation to subjective tiredness is unknown. Thus, in the HAMD-6, fatigue/ fatigability is dispersed rather than a single entity and the term tiredness is not used.

The visual analogue scale has a long history in the quantification of subjective experience (Cowdry and Gardner, 1991). We developed a 6-item version (VAS-6) to complement the HAMD-6 - the traditional $10 \mathrm{~cm}$ line with anchor points at either end. The 6th item, the objective quantification of retardation, did not align with the VAS schema - thus, we developed anchor points concerning subjective experience of the ability to concentrate. In a large naturalistic study, we established the VAS-6 as a valid subjective measure of the core aspects of depression as measured by the objective HAMD-6 (May and Pridmore, 2019).

We found no report focusing on the impact of TMS on subjective tiredness in MDD. Kakuda et al. (2016) studied TMS in the treatment of chronic fatigue syndrome and reported a reduction in fatigue, using a VAS which they developed for that purpose. Fitzgibbon et al. (2018) studied TMS in fibromyalgia and reported a reduction in fatigue using a Multidimensional Fatigue Inventory.

The aim was to understand: 1) whether VAS-tiredness is a valid measure of a feature/s of MDD, 2) whether TMS treatment reduces the subjective tiredness (measured by VAS-tiredness) occurring in MDD, 3) the rate of change of tiredness for different types of TMS treatment. 


\section{Method}

\section{Participants}

We provide TMS to inpatients at a private psychiatric hospital in an Australian capital city. This naturalistic study was approved by The Melbourne Clinic Research Ethics Committee. On admission patients give written approval for their deidentified data to be analysed and published anonymously.

We provide two TMS treatment programs: 1) an 'Acute course' for individuals experiencing acute MDD - comprising 20 daily treatment sessions (with a 2 day rest after the 10th day), and 2) a 'Relapse prevention' (RP) course (Pridmore et al., 2018; Pridmore and May, 2018) - which is similar to what has been termed "maintenance" TMS (Fitzgerald et al., 2013) - this is provided to people with a history of frequent relapse - comprising 5 TMS sessions over 3 days, repeated according to clinical need - not more frequently than 4 weekly and usually at 5-6 week intervals. All participants must meet the diagnostic criteria for MDD according to DSM-5 criteria (Association, 2013).

\section{Measures}

Our standard assessment includes pre- and post-treatment HAMD-6, VAS-6 and Clinical Global Impression for Severity (CGI-S) (Guy, 1976). The 6-item Hamilton Depression Rating Scale (HAMD-6) (O'Sullivan et al., 1997) is superior to the HAMD-17 in terms of transferability, scalability and responsiveness (Hooper and Bakish, 2000; Timmerby et al., 2017) and is unidimensional (Kieslich da Silva et al., 2018). It has been successfully applied in MDD, bipolar depression and depression with mixed features. It is described as "reliable, valid and sensitive to change" and "a clinically useful measure" (Lee et al., 2017). The CGI-S is an assessment of the severity of symptoms of mental disorders, made by a trained clinician on the basis of clinical experience in psychiatry (Busner and Targum, 2007; Guy, 1976). Ratings are from 1 (Normal, not at all ill) through to 7 (Among the most extremely ill patients). It is reliable and widely used (Pinna et al., 2015). The VAS-6 corresponds to the items from the HAMD-6 (May and Pridmore, 2019). For this study we also administered pre- and post-treatment a VAS - a $10 \mathrm{~cm}$ line with the anchor points: "Most tired possible" and "Not at all tired", which was termed 'VAS-tiredness'.

\section{Procedure}

In both the Acute and RP courses, TMS is provided to the left prefrontal cortex, at $110 \%$ motor threshold, $10 \mathrm{~Hz}$ stimulation, 4 second trains, 15 second intertrain intervals, 70 trains per day. Participants completed the measures pre- and post-treatment. 


\section{Data Analysis}

Repeated measures ANOVA were used to compare pre- and post-treatment scores by group (Acute or RP) for the variables of interest. Mixed modelling using random effects was used to understand any influence of repeated courses and group on the change in VAS-tiredness. Pearson correlations were used to analyse associations between variables. Change scores were calculated as: pre score minus post score. All data were entered into SPSS 25 for analysis.

\section{Results}

\section{Participant characteristics}

135 Acute patients and $72 \mathrm{RP}$ patients received treatment. There were 52 participants with tiredness data in the Acute group and 26 in the RP group, totalling 78 participants hereon used in data analyses. Of the participants in the Acute group with tiredness data, the mean age was 41.5 years $(\mathrm{SD}=14.0)$ with 43 female $(83 \%)$. In the RP group the mean age was 45.5 years $(\mathrm{SD}=15.0)$ with 23 female (88.5\%). Of the Acute group, 12 patients received more than one course, for a total of 86 acute courses. Of the RP group, all received multiple courses for a total of $266 \mathrm{RP}$ courses.

\section{Change in symptoms following treatment}

Table 1 shows the means and standard deviations of the pre- and posttreatment scores for the VAS-tiredness, HAMD-6, VAS-6, and CGI-S. There was a significant decrease in tiredness scores following TMS treatment, main effect of time $F(1,350)=147.3, p<.001, \eta^{2}=.30$, a significant difference in tiredness between the two groups, $F(1,350)=10.4, p<.001, \eta^{2}=.03$ and a time by group interaction, $F(1,350)=17.4, p<.001, \eta^{2}=.05$. This showed that the Acute group had a higher tiredness score than the RP group at the beginning of treatment, but they had similar tiredness levels following treatment, Figure 1. There were also significant decreases in HAMD-6 and VAS-6 total scores and for the CGI-S following TMS treatment. The same interaction effect was found for these three measures, with pre-treatment scores being higher in the Acute group but having similar levels to the RP group posttreatment. Results of the mixed modelling showed there was no effect of the number of repeat treatments received on the change in VAS-tiredness score $(p=.49)$.

\section{Associations between tiredness and other depression measures}

Associations between tiredness and other depression measures, both pre- and post-treatment, are shown in Table 2. As expected, in the whole sample VAStiredness before and after treatment correlated with the corresponding HAMD- 
6, VAS-6 and CGI-S. Change in these scores were also correlated with change in VAS-tiredness in the whole sample. Considering the groups separately, the RP group showed the same significant associations as the whole sample, but the Acute group had fewer significant associations, particularly at pre-assessment.

\section{Rate of change for Acute and RP treatment}

We were interested to investigate the effect of individual treatments and time on tiredness levels (as measured using VAS-tiredness). The Acute treatment takes 22 days and is comprised of 20 treatments (with a two day respite after 10 days). The VAS-tiredness pretreatment score was 7.5 and the posttreatment score was 4.9, giving a difference of 2.6. This gives a reduction in tiredness units per treatment of 0.13 , and per day of 0.12 . The RP treatment is 5 treatment sessions over 3 days. VAS-tiredness scores pre- and posttreatment were 6.0 and 4.7 - giving a difference of 1.3 . This gives a reduction in tiredness units per treatment of 0.26 and per day of 0.5 .

\section{Discussion}

The aims of this study were to explore whether VAS-tiredness was a valid measure of a feature/s of MDD and whether TMS treatment reduced the subjective tiredness (measured by VAS-tiredness) occurring in MDD. We found the VAS-tiredness measure showed consistent medium to large correlations with the other depression measures, notably the clinician rated HAMD-6 and corresponding self-report VAS-6, in the whole group. Change in tiredness was association with change in depression. We also found selfreported tiredness decreased in participants treated with TMS. This indicates that tiredness as measured using the VAS-tiredness is a valid feature of depression and this symptom is reduced through TMS treatment.

This study also aimed to explore the rate of change in tiredness for different treatment schedules. The change per treatment for the RP group was almost twice than that for the Acute group. This is consistent with the Acute group being much more severely depressed on admission (HAMD-6 of 10.8, compared to the RP group, 6.9). For the RP group, the change per day is almost twice than the change per treatment. Thus, when the treatment is doubled, the improvement is almost doubled. This is consistent with the new concept of "accelerated treatment" - the administration of multiple treatment sessions per day (Rachid, 2019).

The limitations of this study are that it was not blinded or placebo controlled, however, it is doubtful that introducing such conditions would have influenced the outcome. This was an observational study in which a large number of real patient experiences were collected and analysed with appropriate statistical methods. It may have been beneficial to record VAS- 
tiredness more frequently - but, this would have added to the patient experience of being an 'experimental subject'. Instead, we examined VAStiredness pre- and post-treatment along with the standard instruments we have applied in a routine manner for some years.

Before treatment, the VAS-tiredness was higher for Acute course (7.5) than RP course patients (6.0). This was expected. Tiredness correlates with levels of depression. Acute course patients are suffering acute MDD which has not responded to (usually) long periods of psychotherapy and pharmacological treatment, whereas the RP patients have recently (usually, about 45 weeks ago) been in or very close to remission. Post-treatment VAStiredness was very similar for Acute course (4.9) and RP course (4.7) patients. Again, this was expected. While the Acute course patients began with a higher VAS-tiredness, they received four times more treatment.

Remission is operationalized as a HAMD- 6 score of $\leq 4$ (Bech et al., 2007). Thus, both groups achieved remission and the VAS-tiredness scores could be expected to be similar. Bandelow et al. (2006) state that CGI-S score of 2 or less indicates remission. Pre-treatment, overall, Acute and RP groups are greater than moderately ill. After treatment overall and RP group are in remission, and at 2.1, the Acute group is very close to remission.

It was also found that correlations between the VAS-tiredness and other measures were non-significant or low only for the Acute group pre-treatment for the HAMD-6 and CGI-S, but moderate to strong post-treatment in this group. In the RP group correlations were moderate to strong both pre- and post-treatment. This phenomenon has been found in our previous research comparing self-report on the VAS-6 and clinician report on the HAMD-6 (May and Pridmore, 2019). This finding likely relates to two possibilities. New patients in the Acture group may not yet be familiar with their own depression symptoms, with their knowledge improving during treatment resulting in stronger clinician and self-report correlations post-treatment. The RP group in contrast are familiar with depression symptoms given their various prior treatments and episodes of depression. Alternatively, new patients may not be well known to staff and hence there may be a discrepancy in their initial clinical ratings which reduces over time as they remain in hospital for their Acute treatment and become better known to staff.

This study would have been problematic if TMS caused tiredness. We found one mention of "excessive tiredness" as a side-effect in one large review (Rossi, 2009) - but we could not locate the report of this side effect within the indicated journal. Tiredness is not mentioned as a side-effect in authoritative textbooks (Pascual-Leone et al., 2002; George and Belmaker, 2007). A recent review of "accelerated" TMS (Rachid, 2019) (in which many treatment sessions are provided in one day) also makes no mention of tiredness as a side-effect. Authors reporting the impact of TMS on the 
symptoms of chronic fatigue syndrome (Kakuda et al., 2016) and fibromyalgia (Fitzgibbon et al., 2018) identified reductions in fatigue, rather than increases. Thus, we found no convincing evidence indicating TMS causing tiredness.

Nevertheless, during this study, occasional patients have reported, in unstructured clinical conversations, that treatment was associated with midcourse increased tiredness. This tiredness was not cumulative and prominent tiredness is not apparent in the latter stages of courses of 20 treatments. We conceptualized this occasional mid-course tiredness as a feature of the recovery process. It is possible that should the pre-treatment mental state feature high arousal (or a constellation of other symptoms) producing an inability to relax, when the mental state improves (with treatment) the patient may be more able to experience tiredness (and restorative sleep). We are also considering whether some acutely depressed individuals lack the necessary drive for activity, but as drive improves (with treatment) they perhaps 'make up for lost time', do more, and experience more natural tiredness/sleep.

In this study we rated tiredness at intake and discharge, but not during the course of treatment. To explore the observation of occasional mid-course tiredness we are planning a future study incorporating daily rating of VAStiredness and other complementary tools. We are also planning to explore the impact (if any) of TMS on irritability, which has recently been described as an overlooked feature of MDD (Jha et al., 2019).

In conclusion, VAS-tiredness is a subjective measurement which is additional to the items of the VAS-6, which is based on and is complementary to the objective HAMD-6. VAS-tiredness ratings correlate with the total HAMD6 and VAS- 6 ratings and are a potentially useful addition in the study of patients experiencing MDD.

\section{Funding}

This research did not receive any specific grant from funding agencies in the public, commercial, or non-for-profit sectors.

\section{Author Contributions}

All authors listed have made a substantial, direct and intellectual contribution to the work, and approved it for publication.

\section{Conflict of Interest Statement}

The authors declare that the research was conducted in the absence of any commercial or financial relationships that could be construed as a potential conflict of interest. 


\section{REFERENCES}

American Psychiatric Association (2013). Diagnostic and Statistical Manual of Mental Disorders: DSM-5. 5th edn.

Bandelow, B., D. S. Baldwin, O. T. Dolberg, H. F. Andersen, and D. J. Stein (2006). "What Is the Threshold for Symptomatic Response and Remission for Major Depressive Disorder, Panic Disorder, Social Anxiety Disorder, and Generalized Anxiety Disorder?," Journal Clinical Psychiatry 67(9): 1428-1434.

Bech, P., M. Lunde, G. Bech-Andersen, L. Lindberg, and K. Martiny (2007). "Psychiatric Outcome Studies (POS): Does Treatment Help the Patients? A Popperian Approach to Research in Clinical Psychiatry: 25th Anniversary Report from the Psychiatric Research Unit, Frederiksborg General Hospital, Denmark," Nordic Journal Psychiatry 61(S46): 4-34.

Busner, J., and S. D. Targum (2007). "The Clinical Global Impressions Scale: Applying a Research Tool in Clinical Practice," Psychiatry (Edgmont) 4(7): 28-37.

Chu, A. L., J. Stochl, G. Lewis, S. Zammit, P. B. Jones, and G. M. Khandaker (2019). "Longitudinal Association between Inflammatory Markers and Specific Symptoms of Depression in a Prospective Birth Cohort," Brain, Behavior, and Immunity 76: 74-81.

Cohen, J. (1997). “Assessment and Treatment of Dysthymia. The Development of the Cornell Dysthymia Rating Scale," European Psychiatry 12(4): 190-193.

Cowdry, R. W., D. L. Gardner, K. M. O'Leary, E. Leibenluft, and D. R. Rubinow (1991). "Mood Variability: A Study of Four Groups," American Journal Psychiatry 148(11): 1505-1511.

Crawford, J. R., and J. D. Henry (2004). "The Positive and Negative Affect Schedule (PANAS): Construct Validity, Measurement Properties and Normative Data in a Large Non-Clinical Sample," British Journal Clinical Psychology 43(3): 245-265.

Crowe, E., M. Daly, L. Delaney, S. Carroll, and K. M. Malone (2018). "The IntraDay Dynamics of Affect, Self-Esteem, Tiredness, and Suicidality in Major Depression," Psychiatry Research. https://doi.org/10.1016/j.psychres.2018.02.032.

Fitzgerald, P. B., N. Grace, K. E. Hoy, M. Bailey, and Z. J. Daskalakis (2013). “An Open Label Trial of Clustered Maintenance rTMS for Patients with Refractory Depression," Brain Stimulation 6(3): 292-297.

Fitzgibbon, B. M., K. E. Hoy, L. A. Knox, E. K. Guymer, G. Littlejohn, D. Elliot, et al. (2018). "Evidence for the Improvement of Fatigue in Fibromyalgia: A 4-week Left Dorsolateral Prefrontal Cortex Repetitive Transcranial Magnetic Stimulation Randomized-Controlled Trial," European Journal Pain 22(7): 1255-1267.

George, M. S., and R. H. Belmaker (eds.) (2007). Transcranial Magnetic Stimulation in Clinical Psychiatry. Arlington, VA: American Psychiatric Publishing.

Ghanean, H., A. K. Ceniti, and S. H. Kennedy (2018). "Fatigue in Patients with Major Depressive Disorder: Prevalence, Burden and Pharmacological Approaches to Management," CNS Drugs 32(1): 65-74.

Grape, H. E., K. N. Solbrække, M. Kirkevold, and A. M. Mengshoel (2017). "Tiredness and Fatigue during Processes of Illness and Recovery: A Qualitative Study of Women Recovered from Fibromyalgia Syndrome," Physiotherapy Theory Practice 33(1): 31-40.

Guy, W. (1976). Assessment Manual for Psychopharmacology. Revised. DHEW Publication ABM 76-366. Washington, DC: US Government Printing Office. 
Hamilton, M. (1960). “A Rating Scale for Depression," Journal Neurology Neurosurgery Psychiatry 23(1): 56-62.

Hooper, C. L., and D. Bakish (2000). "An Examination of the Sensitivity of the SixItem Hamilton Rating Scale for Depression in a Sample of Patients Suffering from Major Depressive Disorder," Journal of Psychiatry \& Neuroscience 25(2): 178184.

Jha, M. K., A. Minhajuddin, C. South, A. J. Rush, and M. H. Trivedi (2019). "Irritability and Its Clinical Utility in Major Depressive Disorder: Prediction of Individual-Level Acute-Phase Outcomes Using Early Changes in Irritability and Depression Severity," American Journal of Psychiatry 176(5): 358-366.

Kakuda, W., R. Momosaki, N. Yamada, and M. Abo (2016). "High-Frequency rTMS for the Treatment of Chronic Fatigue Syndrome: A Case Series," Internal Medicine 55(23): 3515-3519.

Katon, W., M. Von Korff, E. Lin, G. Simon, E. Walker, J. Unützer, et al. (1999). "Stepped Collaborative Care for Primary Care Patients with Persistent Symptoms of Depression: A Randomized Trial," Archives of General Psychiatry 56(12): 1109-1115.

Kieslich da Silva, A., M. Reche, A. F. d. S. Lima, M. P. A. Fleck, E. Capp, and F. M. Shansis (2019). "Assessment of the Psychometric Properties of the 17- and 6item Hamilton Depression Rating Scales in Major Depressive Disorder, Bipolar Depression and Bipolar Depression with Mixed Features," Journal of Psychiatric Research 108: 84-89.

Kroenke, K., R. L. Spitzer, and J. B. Williams (2001). "The PHQ-9: Validity of a Brief Depression Severity Measure," Journal General Internal Medicine 16(9): 606-613.

Lee, C. P., C. Y. Liu, and C. I. Hung (2017). "Psychometric Evaluation of a 6-Item Chinese Version of the Hamilton Depression Rating Scale: Mokken Scaling and Item Analysis," Asia Pacific Psychiatry 9(3): e12287.

May, T., and S. Pridmore (2019). "Impact of Transcranial Magnetic Stimulation on the Symptom Profile of Major Depressive Episode," Australasian Psychiatry 27(3): 297-301.

May, T., and S. Pridmore (2019). "A Visual Analogue Scale Companion for the 6Item Hamilton Depression Rating Scale," Australian Psychologist. https://doi. org/10.1111/ap.12427

Montgomery, S. A., and M. Åsberg (1979). “A New Depression Scale Designed to Be Sensitive to Change," British Journal Psychiatry 134: 382-389.

O'Sullivan, R. L., M. Fava, C. Agustin, L. Baer, and J. F. Rosenbaum (1997). "Sensitivity of the Six-Item Hamilton Depression Rating Scale," Acta Psychiatrica Scandinavica 95(5): 379-384.

Pascual-Leone, A., N. J. Davey, J. Rothwell, E. M. Wasserman, and B. K. Puri (eds.) (2002). Handbook of Transcranial Magnetic Stimulation. London: Arnold.

Pinna, F., L. Deriu, E. Diana, V. Perra, R. P. Randaccio, L. Sanna, et al. (2015). "Clinical Global Impression-Severity Score as a Reliable Measure for Routine Evaluation of Remission in Schizophrenia and Schizoaffective Disorders," Annals General Psychiatry 14: 6.

Pridmore, S., S. Erger, M. Rybak, E. Kelly, and T. May (2018). "Early Relapse (ER) Transcranial Magnetic Stimulation (TMS) in Treatment Resistant Major Depression," Brain Stimulation 11(5): 1098-1102. 
Pridmore, S., and T. May (2018). "Relapse Prevention (RP) TMS," Brain Stimulation 11(6): 1391-1392.

Rachid, F. (2019). "Accelerated Transcranial Magnetic Stimulation for the Treatment of Patients with Depression: A Review," Asian Journal of Psychiatry 40: 71-75.

Rossi, S., M. Hallett, P. M. Rossini, and A. Pascual-Leone (2009). "Safety, Ethical Considerations, and Application Guidelines for the Use of Transcranial Magnetic Stimulation in Clinical Practice and Research," Clinical Neurophysiology 120(12): 2008-2039.

Silverstein, B. (1999). "Gender Difference in the Prevalence of Clinical Depression: The Role Played by Depression Associated with Somatic Symptoms," American Journal Psychiatry 156(3): 480-482.

Stadje, R., K. Dornieden, E. Baum, A. Becker, T. Biroga, S. Bösner, et al. (2016). "The Differential Diagnosis of Tiredness: A Systematic Review," BMC Family Practice 17: 147.

Timmerby, N., J. H. Andersen, S. Søndergaard, S. D. Østergaard, and P. Bech (2017). "A Systematic Review of the Clinimetric Properties of the 6-Item Version of the Hamilton Depression Rating Scale (HAM-D6)," Psychotherapy and Psychosomatics $86(3)$ : 141-149.

Vaccarino, A. L., T. L. Sills, K. R. Evans, and A. H. Kalali (2008). "Prevalence and Association of Somatic Symptoms in Patients with Major Depressive Disorder," Journal of Affective Disorders 110(3): 270-276.

World Health Organization (1992). The ICD-10 Classification of Mental and Behavioural Disorders: Clinical Descriptions and Diagnostic Guidelines. Geneva.

World Health Organization (2018). International Statistical Classification of Diseases and Related Health Problems. 11th rev. edn. Geneva.

Figure 1 Change in tiredness scores for the Acute and Relapse Prevention (RP) groups pre and post treatment, with $95 \%$ Confidence Intervals.

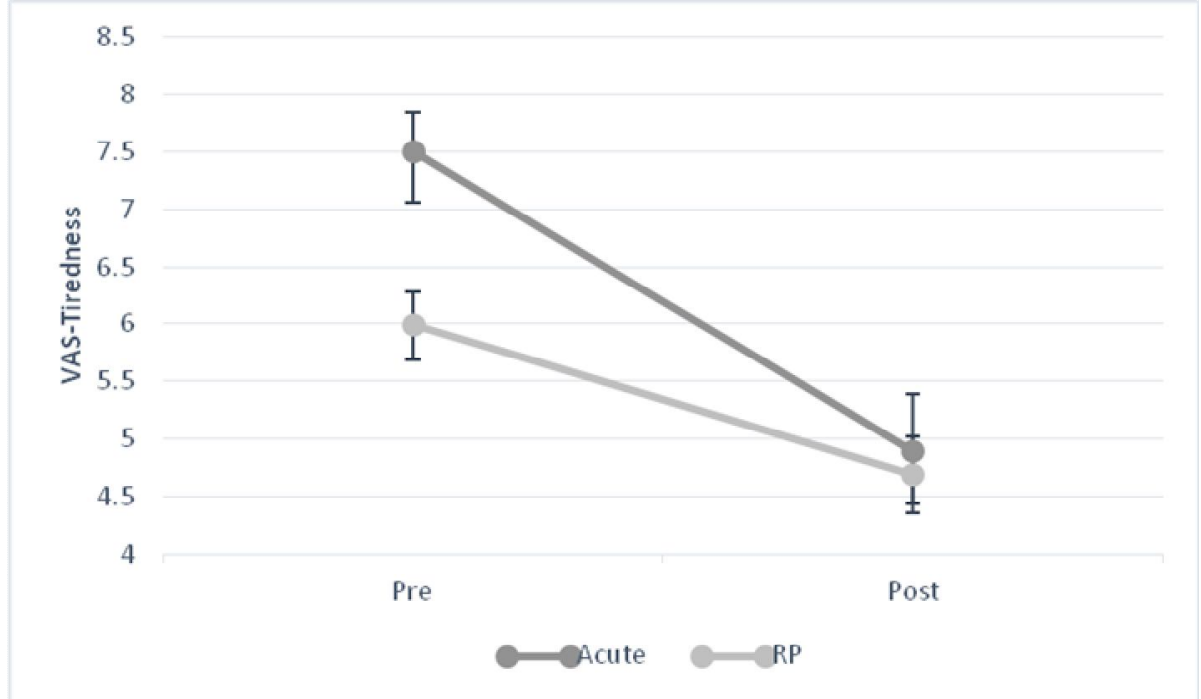


Table 1 Pre post means and standard deviations for the variables of interest

\begin{tabular}{lll}
\hline Measure & Pre & Post \\
\hline Whole group & & \\
$\quad$ Tiredness-VAS $(\mathrm{N}=352)$ & $6.3(2.4)$ & $4.8(2.4)$ \\
HAMD-6 total $(\mathrm{N}=351)$ & $7.8(3.3)$ & $3.4(2.3)$ \\
VAS-6 $(\mathrm{N}=351)$ & $27.1(12.5)$ & $16.7(9.7)$ \\
CGI-S (N=350) & $3.4(1.1)$ & $1.8(0.8)$ \\
Acute group & & \\
Tiredness-VAS $(\mathrm{N}=86)$ & $7.5(1.9)$ & $4.9(2.4)$ \\
HAMD-6 total $(\mathrm{N}=86)$ & $10.8(2.0)$ & $4.0(2.8)$ \\
VAS-6 (N=86) & $38.4(8.8)$ & $20.0(10.4)$ \\
CGI-S (N=84) & $4.3(0.7)$ & $2.1(1.0)$ \\
Relapse Prevention group & & \\
Tiredness-VAS $(\mathrm{N}=266)$ & $6.0(2.5)$ & $4.7(2.5)$ \\
HAMD-6 total $(\mathrm{N}=265)$ & $6.9(3.0)$ & $3.2(2.1)$ \\
VAS-6 (N=265) & $23.4(11.3)$ & $15.7(9.3)$ \\
CGI-S (N=266) & $3.1(1.0)$ & $1.8(0.8)$ \\
\end{tabular}

Table 2 Pearson correlations between VAS-tiredness and HAMD-6, VAS-6 and CGI-I

\begin{tabular}{|c|c|c|c|}
\hline & Whole group & Acute & RP \\
\hline \multicolumn{4}{|l|}{ Pre VAS-Tiredness } \\
\hline Pre HAMD-6 Total $(\mathrm{N}=351,86,265)$ & $.447 * *$ & .158 & $.415^{* *}$ \\
\hline Pre VAS-6 Total $(\mathrm{N}=352,86,266)$ & $.525 * *$ & $.284 * *$ & $.508 * *$ \\
\hline Pre CGI-I $(\mathrm{N}=352,86,266)$ & $.407 * *$ & .056 & $.381 * *$ \\
\hline \multicolumn{4}{|l|}{ Post VAS-Tiredness } \\
\hline Post HAMD-6 Total $(\mathrm{N}=352,86,266)$ & $.406 * *$ & $.463 * *$ & $.390 * *$ \\
\hline Post VAS-6 Total $(\mathrm{N}=351,86,265)$ & $.446^{* *}$ & $.457 * *$ & $.448 * *$ \\
\hline Post CGI-I $(\mathrm{N}=350,84,266)$ & $.348 * *$ & $.396 * *$ & $.335 * *$ \\
\hline \multicolumn{4}{|l|}{ Change in VAS-Tiredness (T2-T1) } \\
\hline Change HAMD-6 Total $(\mathrm{N}=352,86,265)$ & $.372 * *$ & $.213^{*}$ & $.362 * *$ \\
\hline Change VAS-6 Total $(\mathrm{N}=351,86,265)$ & $.421 * *$ & $.354 * *$ & $.384 * *$ \\
\hline Change CGI-I $(\mathrm{N}=350,84,266)$ & $.307 * *$ & .130 & $.314 * *$ \\
\hline
\end{tabular}

$* * \mathrm{p}<.01, * \mathrm{p}<.05$ 